[0212-7199 (2004) 21: 2; pp 79-80] ANALES DE MEDICINA INTERNA Copyright $\odot 2004$ ARAN EDICIONES, S.L.

AN. MED INTERNA (Madrid) Vol. 21, N. $^{\circ} 12$, pp. $79-80,2004$

\title{
Púrpura de Schönlein-Henoch en adultos: estudio de 9 casos
}

A. I. AMEAL GUIRADO, J. MONTES SANTIAGO'

Unidad de Medicina Familiar y Comunitaria. ${ }^{I}$ Servicio de Medicina Interna. Hospital Meixoeiro. Vigo

HENOCH-SHÖNLEIN PURPURA IN ADULTS: A STUDY OF 9 CASES

\section{RESUMEN}

Objetivo: La púrpura de Schönlein-Henoch (PSH) es una vasculitis predominantemente pediátrica de pronóstico generalmente benigno. En adultos puede revestir mayor severidad con mayor frecuencia de afectación renal. Revisamos sus manifestaciones en 9 pacientes durante un periodo de 8 años.

Material y métodos: Revisión retrospectiva de las vasculitis leucocitoclásticas ingresadas en nuestro hospital (periodo 1995-2002), seleccionando los pacientes que cumplían $\geq 3$ de los criterios de Michel para PSH: a) púrpura palpable; b) angina abdominal c) sangrado gastrointestinal; d) hematuria; e) edad >20 años; y f) ausencia de medicación responsable. La incidencia fue de 0,65 casos/100.000 habitantes>15 años, sin predominio estacional. Identificamos 9 pacientes (7 varones, rango de edad: 22-88 años). Hubo púrpura palpable en 100\%, afectación gastrointestinal en $67 \%$, renal en $78 \%$, y articular en $22 \%$. La recuperación fue completa, excepto un caso con insuficiencia renal previa.

Conclusiones: En nuestra serie encontramos una baja incidencia de afectación articular y alta de participación renal con excelente pronóstico.

PALABRAS CLAVE: Púrpura de Schönlein-Henoch. Adultos.
ABSTRACT

Objective: Schönlein-Henoch purpura $(P S H)$ is a predominantly pediatric vasculitis with, generally, a benign prognosis. However, in adults can adquire greater severity and more frequency of renal involvement. We report our experience with 9 cases of this entity.

Material and methods: Retrospective review of the leucocitoclastic vasculitis admitted to our hospital in 1995-2002. Patients fulfilling $\geq 3$ of Michels's criteria for PSH: a) palpable purpura; b) abdominal angina; c) gastrointestinal bleeding; d) hematuria; e) age $>20$ years; $y$ f) absence of responsible medication), were identified. The incidence was 0,65 cases/100.000 inhabitants $>15$ years, without seasonal predominance. We identified 9 patients ( 7 males, age range: 22 to 88 years). There was palpable purpura in 100\%, gastrointestinal involvement in $67 \%$, renal in $78 \%$, and oligoarticular in $22 \%$. Recovery was uneventful, except for a case with previous renal insufficiency.

Conclusions: We found a low frequency of articular involvement and high renal afectation but with an excellent prognosis.

KEY WORDS: Henoch-Schönlein purpura. Adulthood.

Ameal Guirado AI, Montes Santiago J. Púrpura de Schönlein-Henoch en adultos: estudio de 9 casos. An Med Interna (Madrid) 2004; 21: 79-80.

\section{INTRODUCCIÓN}

La primera descripción del síndrome o púrpura de Schönlein-Henoch (PSH) fue hecha en 1837 por Schönlein en un niño con púrpura y artritis. Henoch en 1874 añadió el reconocimiento de la afectación renal e intestinal (1). Sin embargo, probablemente el personaje más famoso al que se atribuye haber padecido dicho síndrome fue Wolfgang Amadeus Mozart, fallecido en 1791. Además de los vómitos y dolores abdominales cólicos, episodios de poliartritis y un exantema verosímilmente purpúrico padecido en la infancia, a este síndrome atribuyen algunos, aunque no sin controversia, la insuficiencia renal terminal que ocasionó su prematura muerte a los 35 años $(2,3)$.

La PSH es una entidad caracterizada por la afectación de piel, articulaciones, tracto gastrointestinal y riñones, teniendo como sustrato histopatológico una vasculitis leucocitoclástica de los pequeños vasos. Considerada como una afección fundamentalmente pediátrica y de buen pronóstico, su presenta- ción en adultos es menos conocida, aunque se sugiere que en estos la evolución puede ser menos benigna (4). En este trabajo se revisan las manifestaciones de PSH en adultos en nuestro hospital en los últimos 8 años.

\section{CASOS APORTADOS}

Se revisaron de forma retrospectiva las historias de los pacientes diagnosticados de vasculitis leucocitoclástica ingresados en nuestro centro en el periodo 1995-2002. Se trata de un hospital terciario de 418 camas que atiende a una población rural y urbana de 154.976 personas $>15$ años, careciendo de los Servicios de Obstetricia y Pediatría. Los pacientes fueron identificados mediante el código 287.0 de la Clasificación Internacional de Enfermedades (CIE 9 MC) y sus datos transferidos a una hoja de recogida de datos. De los 28 casos identificados se seleccionaron los 9 pacientes que cumplían $\geq 3$ de los criterios propuestos por Michel et al. (5), para el diagnóstico de PHS. Dichos criterios son: a) púrpura palpable; b) angina abdominal; c) sangrado gastrointestinal; d) hematuria; e) edad >20 años; y f) ausencia de medi- 
cación que pudiera originar dicho cuadro. Se identificó, además, otro caso en una paciente de 19 años, que se excluyó del presente estudio clínico, aunque sí fue considerado en el análisis epidemiológico.

Encontramos una incidencia de PSH en pacientes de $>15$ años de $1,3 / 100.000$ altas (10/ 78.154) y de 0,65/100.000 habitantes >15 años. Las características clínicas de los pacientes se detallan en la tabla I. De los 9 pacientes, 7 eran varones. El rango de edad fue de 22-88 años. No hubo predominio estacional claro (4 en primavera, 1 en verano, 3 en otoño y 1 en invierno). Un paciente recibió doxiciclina pero no se le consideró el causante porque el cuadro se inició previamente a su administración. En 4 casos hubo evidencia de infección previa o concomitante: 2 infecciones respiratorias, 1 infección urinaria y 1 apendicitis. Todos presentaron púrpura palpable. Existieron manifestaciones gastrointestinales en 6 pacientes (2: dolor abdominal, 1: dolor y vómitos, 1: dolor y rectorragia, 1: vómitos, 1 vómitos y rectorragia). Hubo afectación renal en 7 pacientes (4: hematuria, 2: hematuria e insuficiencia renal, 1: insuficiencia renal). En sólo 2 pacientes hubo afectación articular (1: monoartritis de tobillo, 1: artritis bilateral de tobillos). Respecto a la analítica, en 2 casos (22\%) se halló leucocitosis ( $>10.000$ leucocitos $\left.\mathrm{x} 10^{9} / \mathrm{L}\right)$, en 2 , anemia $(<12 \mathrm{~g} / \mathrm{dL})$, y en 2 , trombopenia $(<140.000$ plaquetas $x 10^{9} / \mathrm{L}$ ), que fueron transitorias. En 2 de los 7 casos en que se realizó biopsia dérmica se encontró depósito de IgA. Se hallaron niveles de IgA sérica elevados en 6 de los 7 pacientes en que se determinaron.

\section{TABLA I}

CARACTERÍSTICAS CLÍNICAS DE LA PÚRPURA DE SCHÖNLEIN-HENOCH EN 9 PACIENTES ADULTOS

\begin{tabular}{llr}
\hline Manifestaciones clínicas & Casos & $\%$ \\
\hline Púrpura palpable & 9 & 100 \\
$\begin{array}{l}\text { Manifestaciones articulares } \\
\quad \text { (oligoarticular) }\end{array}$ & 2 & 22 \\
Manifestaciones gastrointestinales & 6 & 67 \\
$\quad$ Dolor abdominal & 4 & \\
Vómitos & 3 & \\
$\quad$ Rectorragias & 2 & 78 \\
Manifestaciones renales & 7 & \\
$\quad$ Hematuria & 6 & \\
Insuficiencia renal & 3 (1 previa) & \\
\hline
\end{tabular}

\section{DISCUSIÓN}

Hemos revisado las características clínicas y evolutivas de pacientes diagnosticados de PSH por los criterios de Michel (5). Con dichos criterios, diseñados para distinguir la PSH de la vasculitis por hipersensibilidad se realiza el diagnóstico de PSH con un $87,1 \%$ de sensibilidad (5). Nuestra serie estudia pacientes hospitalizados por dicho cuadro y, por tanto, presumiblemente con un sesgo de gravedad. Aún así obtuvimos una incidencia de 1,3/100.000 pacientes ingresados y de $0,65 / 100.000$ habitantes $>15$ años, la cual es algo superior a la incidencia comunicada de 0,03-0,35/100.000 para pacientes adultos con PSH (6). A diferencia de otras series no encontramos un patrón estacional de presentación definido (7).

Clásicamente, se consideraba a la PSH como la vasculitis más frecuente de la edad pediátrica, con un curso generalmente benigno y autolimitado $(4,7)$. Sin embargo, algunos pacientes pueden presentar una forma más grave, con secuelas a largo plazo. Así, en una serie con seguimiento a los 24 años se detectó insuficiencia renal en hasta el $35 \%$ de los pacientes que padecieron una $\mathrm{PSH}$ grave en la infancia (8).

Las características y evolución de dicha entidad en adultos es poco conocida. No obstante, en los últimos años vienen publicándose observaciones que sugieren que, en ellos, puede representar un cuadro clínico más severo que en los niños, fundamentalmente en relación con una mayor frecuencia de afectación renal $(7,9)$. En nuestra serie se objetivó dicha afectación hasta en el $78 \%$ de pacientes. Sin embargo, la evolución a largo plazo fue buena y excepto una paciente en la que se había demostrado una glomerulonefritis previa y que ulteriormente precisó trasplante renal, en el resto, la hematuria e insuficiencia renal desaparecieron totalmente.

Es de destacar que la afectación articular, que en algunas de las series adultas publicadas se aproxima al 100\% (9), sólo se encontró en el $22 \%$ de nuestros pacientes, más en consonancia con el $24-39 \%$ de otras series $(7,10)$. Ello debe tenerse en cuenta ya que puede dificultar en adultos el reconocimiento de esta entidad al faltar tan clásico indicio. En el $86 \%$ de casos en que se determinó, encontramos aumento sérico de $\operatorname{IgA}$ lo que quizá, como se describe clásicamente, podría ser un dato útil, si bien en otras series esto se halló en menos del $10 \%$ (4).

En resumen, presentamos nuestra experiencia con 9 casos de PSH en los que se encontró una baja incidencia de afectación articular y alta de afectación renal pero con buena recuperación ulterior.

\section{Bibliografía}

1. Blanco R, Rodríguez-Valverde V, Mata-Arnaiz C, Martínez-Taboada VM. Síndrome de Schönlein-Henoch. Rev Esp Reumatol 2000; 27:5465.

2. Landon HCR. El último año de Mozart. Madrid: Siruela, 1988.

3. Montes Santiago J. El coleccionista de cráneos célebres. Recorrido patobiográfico en busca de los cráneos perdidos de Bach, Haydn, Mozart, Beethoven, Goya y el cerebro de Einstein. Salamanca: Graficesa, 2002. p. 74-77.

4. Blanco Alonso R, Martínez-Taboada VM. Vasculitis leucocitoclástica. En: Tratado de Reumatología. Pascual Gómez, E, Rodríguez Valverde V, Carbonell Abelló J, Gómez-Reino Carnota JJ. eds. Madrid: Arán, 1998; p. 683-697.

5. Michel BA, Hunder GG, Bloch DA, Calabrese LH. Hypersensitivity vasculitis an Henoch-Schönlein purpura: a comparison between the two disorders. J Reumatol 1992; 19: 721-8.

6. Watts RA, Carruthers DM, Scott DG. Epidemiology of systemic vascu-

litis: changing incidence or definition? Semin Arthitis Reum 1995; 25: 28-34.

7. Blanco R, Martínez-Taboada VM, Rodríguez-Valverde V, GarcíaFuentes M, González-Gay MA. Henoch-Schönlein purpura in adulthood and childhood. Two different expressions of the same syndrome. Arthritis Reum 1997; 40: 859-64.

8. Ronkainen J, Nuutinen M, Koskimies O. The adult kidney 24 years after childhood Henoch-Shönlein purpura: a retrospective study. Lancet 2002; 360: 666-70.

9. Uthman I, Kassak K, Nasr FW. Henoch-Schönlein purpura in adulthood and childhood: comment on the article by Blanco et al. Arthritis Reumatism 1998; 41: 1518

10. Mills JA, Michel BA, Bloch DA, Calabrese LH, Hunder GG, Arend WP, et al. The American College of Reumathology 1990 criteria for the classification of Henoch-Schönlein purpura. Arthritis Reumat 1990; 33: 1114-21. 